\title{
The Chest Pain Choice trial: a pilot randomized trial of a decision aid for patients with chest pain in the emergency department
}

\author{
Meghan A Pierce1,2, Erik P Hess*1,2, Jeffrey A Kline³, Nilay D Shah1,4, Maggie Breslin1,5, Megan E Branda1,6, \\ Laurie J Pencille1, Brent R Asplin², David M Nestler2, Annie T Sadosty², lan G Stiell7, Henry H Ting 1,8 and \\ Victor M Montori 1,4,9
}

\begin{abstract}
Background: Chest pain is a common presenting complaint in the emergency department (ED). Despite the frequency with which clinicians evaluate patients with chest pain, accurately determining the risk of acute coronary syndrome (ACS) and sharing risk information with patients is challenging. The aims of this study are (1) to develop a decision aid (CHEST PAIN CHOICE) that communicates the short-term risk of ACS and (2) to evaluate the impact of the decision aid on patient participation in decision-making and resource use.
\end{abstract}

Methods/Design: This is a protocol for a parallel, 2-arm randomized trial to compare an intervention group receiving CHEST PAIN CHOICE to a control group receiving usual ED care. Adults presenting to the Saint Mary's Hospital ED in Rochester, MN USA with a primary complaint of chest pain who are being considered for admission for prolonged ED observation in a specialized unit and urgent cardiac stress testing will be eligible for enrollment. We will measure the effect of CHEST PAIN CHOICE on six outcomes: (1) patient knowledge regarding their short-term risk for ACS and the risks of radiation exposure; (2) quality of the decision making process; (3) patient and clinician acceptability and satisfaction with the decision aid; (4) the proportion of patients who decided to undergo observation unit admission and urgent cardiac stress testing; (5) economic costs and healthcare utilization; and (6) the rate of delayed or missed ACS. To capture these outcomes, we will administer patient and clinician surveys after each visit, obtain video recordings of the clinical encounters, and conduct 30-day phone follow-up.

Discussion: This pilot randomized trial will develop and evaluate a decision aid for use in ED chest pain patients at low risk for ACS and provide a preliminary estimate of its effect on patient participation in decision-making and resource use.

Trial registration: Clinical Trials.gov Identifier: NCT01077037

\section{Background}

Over 6 million adults present with chest pain in US emergency departments (EDs) each year, making chest pain the second most common presenting complaint [1]. Despite the frequency with which clinicians evaluate patients with chest pain, reliably detecting patients with acute coronary syndrome (ACS) remains a diagnostic dilemma. Data from U.S. EDs suggest that $2.1 \%$ of

* Correspondence: hess.erik@mayo.edu

1 Knowledge and Encounter Research Unit, Mayo Clinic, Rochester, Minnesota, USA

Full list of author information is available at the end of the article patients with acute myocardial infarction and $2.3 \%$ with unstable angina are missed [2]. A missed diagnosis of ACS may have substantial medical and legal implications [3]. As a result, clinicians initiate formal diagnostic testing for ACS at a very low risk threshold [4]. High sensitivity is ensured at the expense of specificity, resulting in large numbers of very low risk patients being admitted to ED observation units for urgent cardiac stress testing, increased likelihood of false positive stress testing, excessive exposure to ionizing radiation [5], and significant cost to the healthcare system. 
Risk communication in a busy ED setting is also a challenge. When a patient presents to the ED with chest pain and the initial evaluation for non-cardiac etiologies is unrevealing, the focus of the evaluation shifts to estimating a patient's risk for ACS. Without the assistance of decision support tools, clinicians are challenged to precisely quantify risk and often resort to terms such as "low," "rare," or "uncommon" when communicating risk to patients. One recent study reported that patients often overestimate the level of potential harm when verbal descriptors are used to communicate risk [6]. To increase patient understanding of risk and optimize knowledge transfer between physicians and patients, we will develop and evaluate a decision aid that includes a precise estimate of the short-term risk for ACS described using prose phrases, numbers, and a pictograph to account for patients' preferred mode of understanding numerical information.

To date, the authors are unaware of any published research investigating the specific role and impact of shared decision making in patients at low risk for ACS. We hypothesize that patients infrequently participate in the decision to undergo prolonged observation and urgent cardiac stress testing and that the use of a decision aid to communicate both the short-term risk of ACS and the risks of ionizing radiation exposure will increase patient involvement in the decision making process, lead to a preference-based decision, and safely decrease resource use. To test our hypothesis, we will accomplish the following specific aims: (1) develop and refine CHEST PAIN CHOICE, a decision aid to communicate the shortterm risk for an ACS and the available management options and (2) test the effect of CHEST PAIN CHOICE, in a randomized clinical trial, on patient knowledge, the quality of the decision making process, patient safety, and resource use.

\section{Methods \\ Design}

To evaluate the decision aid, we will conduct a patientlevel single center pilot randomized trial. The Mayo Clinic Institutional Review Board has approved all study procedures.

\section{Setting}

Patients and emergency physicians will be recruited from the Saint Marys Hospital ED at the Mayo Clinic, a tertiary care academic ED with an annual census of 73,000 patient visits. The trial will be conducted in the flow of routine patient care. This approach will enable us to test how the decision aid performs in the real-world setting.

\section{Participants \\ Inclusion criteria}

Eligible providers will include clinicians (attending physicians, supervised residents, nurse practitioners and phy- sician assistants) caring for patients with chest pain. Eligible patients will include adults ( $>17$ years of age) presenting to the ED with a primary complaint of non-traumatic chest pain who are being considered for admission to the ED observation unit for urgent cardiac stress testing.

\section{Exclusion criteria}

The trial will exclude patients with an initial cardiac troponin $\mathrm{T}$ value above the $99^{\text {th }}$ percentile reference limit (Roche Diagnostics [Basel, Switzerland]; 99th percentile $<0.01 \mathrm{ng} / \mathrm{mL}$; lower limit of detection $0.01 \mathrm{ng} / \mathrm{mL} ; 10 \%$ coefficient of variation $0.035 \mathrm{ng} / \mathrm{mL}$ ) [7], known coronary artery disease (at least one $50 \%$ stenosis on cardiac catheterization, electrocardiographic changes [old or not known to be old] indicative of ischemia such as ST-T changes or left bundle branch block, perfusion defects or wall motion abnormalities on exercise, pharmacologic or rest imaging studies, or previous documentation of acute myocardial infarction) [8], cocaine use within 72 hours by the clinician's initial history, and pregnancy. Patients who cannot read English or have, in their clinician's best judgment, major communication barriers such as visual or hearing impairment or dementia that would compromise their ability to give written informed consent (or use the decision aid) will be excluded.

\section{Participant recruitment}

We have previously developed and validated an electronic notification system that is highly sensitive and specific for identifying potentially eligible participants presenting to the ED with chest pain as a primary complaint (sensitivity 97.8\%, 95\% CI 96.1-98.8; specificity 98.3\%, 95\% CI 98.098.5; positive predictive value $68.8 \%$, 95\% CI 65.4-72.9; negative predictive value $99.9 \%, 95 \%$ CI 99.8-100.0) [9]. Shortly after arrival to the ED, a study coordinator will receive electronic notification of potentially eligible participants in real time by both pager and email. In collaboration with the treating physician, the coordinator will confirm eligibility for the trial. The coordinator will then inform patients about the trial and obtain written informed consent. Patients will be consecutively enrolled 6-7 days per week when a study coordinator is available for enrollment.

\section{Interventions}

Decision aid

The CHEST PAIN CHOICE decision aid prototype has been developed through an iterative process involving collaboration between designers and researchers, fieldtesting by patients and clinicians, and revision of the decision aid in response to the feedback provided. We have employed this methodology to develop the prior decision aids STATIN CHOICE [10] (assists providers in discussing the benefits and side effects of statins among patients with diabetes) and OSTEOPOROSIS CHOICE [11] (assists with the decision to take bisphosphonates 
among postmenopausal women at risk for osteoporotic fracture) [12,13].

\section{Selection of a risk calculator for the decision aid}

We recently conducted a systematic review to identify clinical prediction tools of sufficient diagnostic accuracy and methodological quality to consider for incorporation in clinical practice [14]. Of the clinical prediction tools that have been developed for ED patients with potential ACS, only 3 have been prospectively validated and thus could be considered for use in clinical practice - the Global Registry of Acute Coronary Events (GRACE) score, the Thrombolysis in Myocardial Infarction (TIMI) risk score, and the computerized quantitative pretest probability calculator. The GRACE risk score has been validated in only one ED study, and in this study data were not available to calculate a complete risk score in $26 \%$ of patients [15]. Although the TIMI risk score has been validated in over 17,000 ED patients in 5 different countries and can provide a reliable estimate of the risk for a cardiac event within 30 days of the ED visit, it has not undergone impact analysis [16]. The pretest probability calculator developed by Kline et al., unlike the TIMI risk score, has undergone impact analysis, demonstrating its true impact on patient care [17]. The pretest probability calculator was derived from data from 14,796 patients enrolled in the multicenter internet tracking registry of ACS database and prospectively validated in 8,120 ED patients with potential ACS [18]. The pretest probability calculator has since been prospectively validated in a multicenter study of 1,114 patients from 3 different academic EDs [19] and undergone impact analysis in a practical randomized trial [17]. For these reasons, we chose to use this calculator (Figure 1) to inform our decision aid CHEST PAIN CHOICE.

\section{Randomization}

We will use a dynamic allocation procedure described by Pocock and Simon [20] to randomly allocate patients via a computer-generated allocation sequence in a 1:1 concealed fashion to one of two arms: (1) a CHEST PAIN CHOICE arm in which the provider and patient will be provided a printout of the decision aid or (2) a usual care arm in which the provider will discuss the management options in their usual fashion without the decision aid or pretest probability calculator.

Given the limited amount of information that is available during the initial ED encounter and the known association of age and sex with cardiovascular risk, we will dynamically stratify by age and sex in the allocation process to ensure that the treatment arms are balanced in regard to these factors. To enroll patients in the trial, the study coordinator will complete the following:
- Assess patient eligibility in collaboration with the treating physician and obtain written informed consent from both parties.

- Access a secure website, input key patient characteristics, and obtain from the website interface the arm to which the patient is allocated.

After randomization, outcome assessors and data analysts will remain blind to allocation. In the event that a patient is suspected of being incorrectly enrolled (i.e., it is discovered after randomization that the patient is ineligible for the trial), the study coordinator will present the case to the principle investigator (E.P.H.) for review. The principle investigator, blinded to both allocation arm and patient outcome, will then consider post-randomization exclusion according to published recommendations [21].

The main disadvantage of randomization at the patient level is reducing the risk of contamination in the control group. Providers may be aware of the hypothesis that use of CHEST PAIN CHOICE will increase the quality of the decision-making process, increase patient knowledge, and decrease resource use. However, we anticipate that clinicians will revert to their usual pattern of practice without the calculator -- since access to it is passwordprotected -- and be reluctant to dismiss patients from the ED. We will also monitor for evidence of contamination by reviewing the video recordings of clinician-patient encounters in the control group.

\section{Intervention arm}

For patients randomized to the intervention arm, the study coordinator will review the contents of the decision aid in real time with the clinician immediately before the patient encounter. This approach to delivering the decision aid (with just-in-time training by the study coordinator prior to use) has been effectively used in our prior decision aid studies [22]. We have observed a trend towards superior results when the decision aid is delivered by the clinician compared to researchers delivering the tool prior to the clinical encounter [23].

\section{Usual care}

For patients randomized to the usual care arm, the clinicians will discuss the results of the diagnostic investigations and management options with the patient in that clinician's usual fashion. We will videotape the patientclinician discussion for future review. No researchrelated interventions will be administered.

\section{Outcome measures}

Decision-based outcome measures take into account both the Ottawa Framework for Shared Decision Making [24] and self determination theory [25]. To assess the efficacy of the decision aid, we will measure the effect of CHEST PAIN CHOICE on the following outcomes: (1) patient knowledge regarding their short-term risk for ACS and the risks of radiation exposure; (2) quality of the 


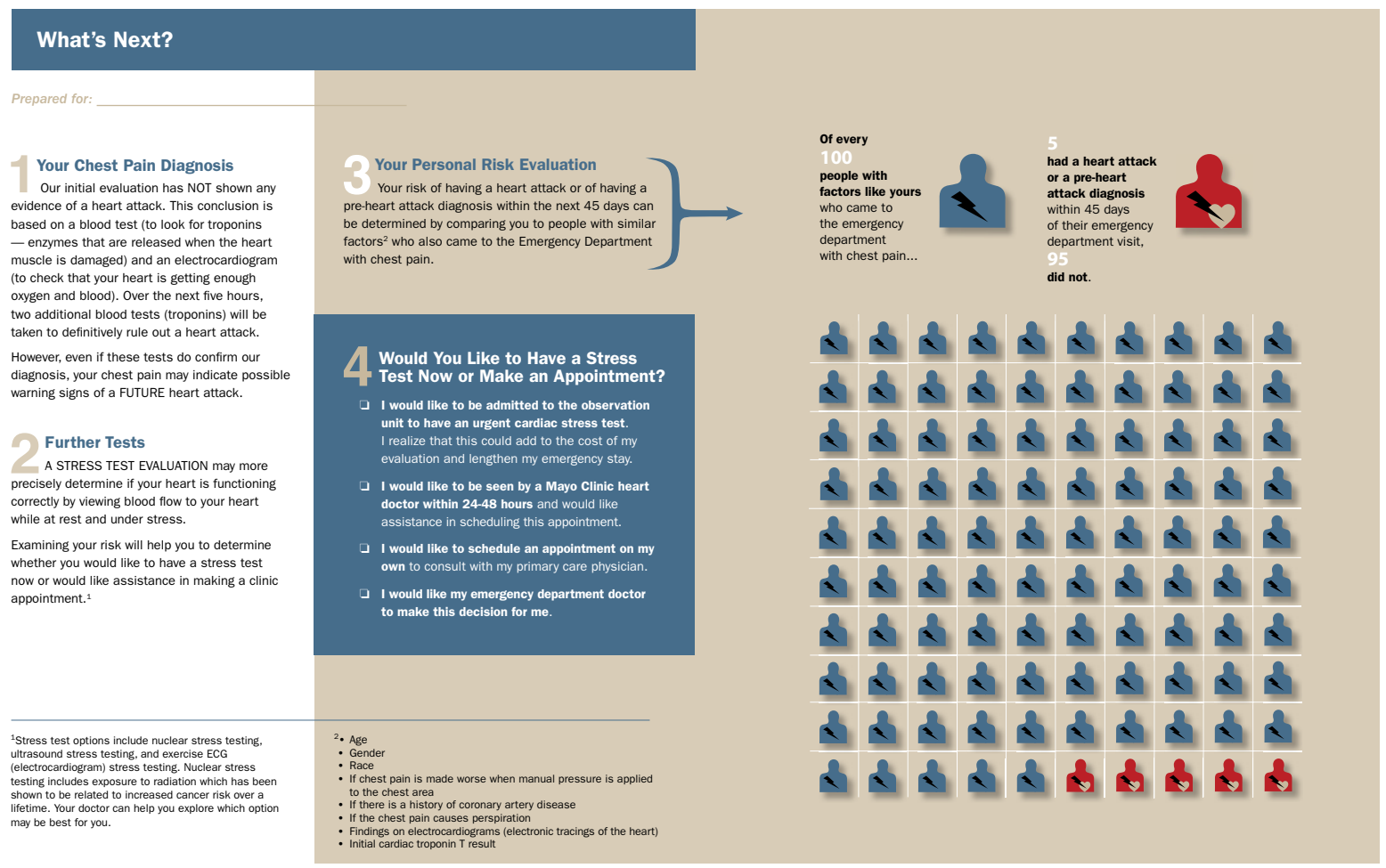

Figure 1 Chest Pain Choice Decision Aid

decision making process for the patient and clinician; and (3) patient and clinician acceptability and satisfaction with the decision aid. We will also assess (4) the proportion of patients who decide to undergo observation unit admission and urgent cardiac stress testing; (5) economic costs and healthcare utilization; and (6) the rate of delayed or missed ACS (primary safety endpoint). The diagnosis of ACS will be considered delayed or missed if the patient was dismissed from the ED without observation unit admission and urgent stress testing and they meet the definition of ACS within 30 days of the ED visit. ACS will be defined as acute myocardial infarction [26], ventricular arrhythmia, cardiogenic shock, bradycardia requiring therapeutic intervention, or death attributed to a cardiac or unknown cause. Two investigators, blinded to allocation, will assess outcomes in all enrolled patients. A third investigator will resolve discordances.

A study coordinator will review the electronic medical record and conduct telephone follow-up for all enrolled patients starting at 30 days. The coordinator will verify the patient's telephone number at the time of enrollment to maximize the fidelity of follow-up. For those who are unable to be contacted by phone, we will use the resources of the Rochester Epidemiology Project [27] to determine outcomes for all Olmsted County residents and the social security death index [28] for patients who live outside of Olmsted County. We will note the success with enrollment and document the rate of 30-day followup.

\section{Data collection}

We will survey patients and clinicians after each visit and videotape the decision aid discussion between the clinician and patient in both the intervention and control groups to capture outcomes. The patient surveys (see Additional files 1 and 2 for complete list of survey questions) will include:

- Items about level of education and income level for descriptive purposes

- Questions regarding the patient's comfort level in understanding different types of numerical information (e.g., fractions, percentages, graphs)

- 7 items to assess patient knowledge, 2 of which address the risks of radiation exposure, 2 that address the available management options, 2 about the potential implications of cardiac stress test results (not informed by content on the decision aid), and one question about the patient's personalized risk for ACS within 45 days of the ED visit. These items were fashioned after similar questions, which have demon- 
strated construct validity and responsiveness to the intervention in previous decision aid trials $[29,30]$.

- 5 items using a 7-point Likert-type scale to assess the patient's perceptions about the amount, clarity and helpfulness of the information, their desire to receive information about other diagnostic options in the same way this information was delivered, and whether they would recommend the way they received information from their clinician during this encounter to other patients. We have used the same items in our previous trials $[11,30]$.

- 10 items from the Trust in Physician scale [31]

- 17 items from the Decisional Conflict Scale [32], which assesses the extent to which the decision was informed, consistent with values, free of pressure, and effective

- Additional questions that inquire about the decision made by the patient during the visit and patient satisfaction with the degree of participation in the decision making process.

The treating clinician will be asked to complete a survey after each study visit (see Additional file 3). The survey includes questions regarding which decision the patient made, the clinician's perception of who made the decision, the clinician's confidence in the patient's understanding of the information, and questions regarding the acceptability of the decision aid.

The discussion between the patient and clinician regarding the risks and benefits of further evaluation (i.e. observation unit admission with stress testing versus urgent follow-up with cardiology or follow-up with the patient's primary physician) and explanation of the CHEST PAIN CHOICE decision aid by the treating clinician will be video recorded to determine the degree of patient participation in the decision making process. We will use the OPTION scale [33] to evaluate the quality of the decision making on the video recordings. The scale enables an observer to quantify the extent to which clinicians involve patients in the decision making process. We have used the OPTION scale with adequate reliability in previous studies [22,34]. Video recordings will also provide information regarding the duration of the encounter (using video time stamps), fidelity of use of the tool, and difficulties encountered while using the decision aid.

We will conduct an economic evaluation of the trial using the Olmsted County Healthcare Expenditure and Utilization Database (OCHEUD). We will limit the analysis to subjects residing in Olmsted County, Minnesota since we have complete data on medical care utilization for this population. OCHEUD is a claims-based database that contains information on medical resource utilization, associated charges, and estimated economic costs for patients receiving care at either the Olmsted Medical Center or the Mayo Clinic and their associated inpatient facilities. Although the services provided represent the clinical practice patterns of Mayo Clinic and Olmsted Medical Center providers, the value of each unit of service has been adjusted to national norms by use of widely accepted valuation techniques. OCHEUD provides an estimated economic cost for each line item in the billing record and allows the aggregation of costs into categories deemed relevant to a particular study. The database serves as a major source of financial information on all patients (i.e., irrespective of payer) and includes line-item detail on date, type, frequency, and billed charge for every good or service provided. We will use data from the OCHEUD database to compare costs and utilization between the intervention and control groups. We will further compare the types of services that were used in each of the groups.

\section{Statistical considerations}

We have sufficient funding to enroll and randomize 200 patients. While this number is insufficient to definitively assess the efficacy of the decision aid and its effect on the proportion of patients who decide to undergo observation unit admission and urgent cardiac stress testing, it is sufficient to determine the following:

- for knowledge gains, assuming the patients in the control group will, on average, answer 4 of the 7 questions correctly, we will have $90 \%$ power to detect a $>25.7 \%$ increase in mean knowledge (to a mean of 5.8 questions answered correctly) in the intervention group, assuming equal variances between the two arms and alpha $=0.05$;

- for the proportion of patients who decide to undergo observation unit admission and stress testing, assuming that at least $90 \%$ of patients in the control group will be admitted to the ED observation unit (null hypothesis), we will have $95 \%$ power to detect a $20 \%$ decrease in the proportion of patients who decide to be admitted to the ED observation unit for stress testing (from 90\% to 70\%; alternative hypothesis) in the decision aid treatment group, with a twosided test at a $5 \%$ significance level.

The feasible sample size is also adequate to provide a plausible effect size to plan a definitive multicenter randomized trial.

We will report baseline characteristics potentially associated with study outcomes (age, gender, education level, numeracy, pretest probability for acute coronary syndrome, smoking history, proportion of patients with hypercholesterolemia, and proportion with a family history of premature coronary artery disease) separately for the intervention and control group. Each factor will be compared using either the Chi-square or Fisher's exact test for categorical variables, depending on which is appropriate for that factor. The continuous factors will be 
compared using the Wilcoxon rank sum test or t-test as appropriate. Any imbalance found within a risk factor will be explored as a possible factor to adjust for when the outcome measures are being analyzed. Any p-value $<0.10$ will be considered a possible imbalance.

Study data will be collected and managed using REDCap electronic data capture tools hosted at the Mayo Clinic [35]. REDCap (Research Electronic Data Capture) is a secure, web-based application designed to support data capture for research studies, providing: 1) an intuitive interface for validated data entry; 2) audit trails for tracking data manipulation and export procedures; 3 ) automated export procedures for seamless data downloads to common statistical packages; and 4) procedures for importing data from external sources.

Continuous variables will be reported as mean ( \pm S.D.) or medians with interquartile ranges as appropriate for the distribution of the data. Categorical data will be presented as percent frequency of occurrence. Variables will be compared using relative (relative risk) or absolute (mean differences) measures of association and their 95\% confidence intervals. To test hypotheses of association, we will use Wilcoxon rank-sum tests to compare medians, and Chi-square or Fisher's exact tests to compare frequencies. All analyses will be based on two-sided tests at a significance level of 0.05 . We will follow the intention to treat principle in our analysis, making every effort to avoid missing data and analyzing patients according to the arm to which they were randomized. All analyses will be conducted using SAS software (SAS institute, Inc, Carey, NC, USA) version 9.1 TS level 1M3.

\section{Discussion}

We have described the design of a clinical trial to determine the effectiveness of a decision aid on patient participation in decision-making and resource use in ED chest pain patients at low risk for ACS. While this is a feasibility trial for a new decision aid, it will provide an estimate of the effect of the decision aid that can be used to inform the design of a larger multicenter trial.

An important reason to conduct feasibility trials of decision aids designed for use in the clinical setting is that the tools have potential to impact the nature and content of the patient-physician discussion and the duration of the clinical evaluation. Patients who present to the ED with chest pain may be in a vulnerable emotional state such that they prefer decisions be made on their behalf rather than actively participate in their medical care. The ED is also the safety net for patients of all levels of education and for those without medical insurance, and we anticipate that there may be knowledge transfer challenges that are unique to this practice setting. When clinicians with different practice patterns and level of experience use the decision aid, they may not find it appropriate to use for some patients or may not use the decision aid appropriately. Therefore, video recording the patient-clinician encounter may provide insight into these potential challenges, the effectiveness of the decision aid, and the nature of the patient-physician interaction in a busy ED environment. The results of this study may guide process changes in trial design, changes in inclusion and exclusion criteria, refinement of the decision aid itself, and data to estimate a sample size for design of a larger efficacy study.

In conclusion, there is an urgent need to develop and evaluate a shared decision making approach to patients presenting with chest pain to the ED. Chest pain is a common complaint in EDs that challenges emergency clinicians to provide an accurate, safe, and efficient diagnosis. Clinicians seem to have a very low risk tolerance and initiate diagnostic testing in patients with a very low pre-test probability of disease. Overtesting for ACS increases the likelihood of false positive stress testing, exposes patients unnecessarily to ionizing radiation, worsens ED overcrowding, and increases the cost of care. Developing and testing a decision aid that transparently communicates the short-term risk for ACS to both clinicians and patients, the potential risks of radiation exposure, and the available management options is a novel approach to solving a common clinical problem in a patient-centered fashion. Our approach differs from prior risk stratification studies of ED patients with potential ACS in that it seeks to enhance patient involvement in the decision making process rather than relying solely on diagnostic accuracy (i.e., ruling out ACS with $100 \%$ sensitivity). We hypothesize that use of CHEST PAIN CHOICE will increase patient knowledge and involvement in the decision making process, decrease physician and patient anxiety, and safely decrease resource use in ED patients with chest pain. We will also continue to develop the expertise of our group in the conduct of decision aid trials in complex real-world settings.

\section{List of abbreviations}

ED: emergency department; ACS: acute coronary syndrome; GRACE: Global Registry of Acute Coronary Events; TIMI: Thrombolysis in myocardial infarction; OCHEUD: Olmsted County Healthcare Expenditure and Utilization Database; REDCap: Research Electronic Data Capture;

\section{Additional material}

Additional file 1 General patient survey.

Additional file $\mathbf{2}$ Wiser choices patient survey (numeracy survey).

Additional file 3 Provider Survey.

Competing interests

The authors declare that they have no competing interests. 


\section{Authors' contributions}

MAP contributed to the design of the study, designed study procedures, and wrote the first draft of the manuscript. EPH made substantial contributions to the conception and design of the study and the study protocol, applied for funding, and made critical revisions to the manuscript. JAK made substantial contributions to the conception and design of the study, developed the pretest probability calculator to inform the decision aid, and made critical revisions to the manuscript. NDS contributed to the conception and design of the study, wrote the economic analysis portion of the manuscript, and provided critical revisions to other sections of the manuscript. MB contributed to the conception and design of the study, designed the decision aid, and provided critical revisions to the manuscript. MEB contributed to the statistical analysis of the study, wrote the statistical section of the manuscript, and provided critical revisions to other sections of the manuscript. LP contributed to the design of the study, designed study procedures, and provided critical revisions to the manuscript. BRA, DMN, ATS, and IGS contributed to the conception and design of the study and made critical revisions to the manuscript. HHT, a cardiologist, provided subspecialty expertise to the conception and design of the study and made critical revisions to the manuscript. VMM made substantial contributions to the conception and design of the study and the study protocol and made critical revisions to the manuscript. All authors approved the final version of this manuscript.

\section{Acknowledgements}

The study is funded by a grant from the Foundation for Informed Medical Decision Making. This funding source had no role in the design of this study and will not have any role during its execution, analyses, interpretation of the data, or decision to submit results.

\section{Author Details}

'Knowledge and Encounter Research Unit, Mayo Clinic, Rochester, Minnesota, USA, 2Division of Emergency Medicine Research, Department of Emergency Medicine, Mayo Clinic, Rochester, Minnesota, USA, ${ }^{3}$ Emergency Medicine Research, Department of Emergency Medicine, Carolinas Medical Center, Charlotte, NC, USA, ${ }^{4}$ Division of Health Care Policy and Research, Department of Health Sciences Research, Mayo Clinic, Rochester, MN USA, 5SPARC Design Studio, Center for Innovation, Mayo Clinic, Rochester, Minnesota, USA, ${ }^{6}$ Division of Biomedical Statistics and Informatics, Department of Health Sciences Research, Mayo Clinic, Rochester, MN, USA, ' Department of Emergency Medicine, University of Ottawa, Ottawa, Ontario, Canada, ${ }^{8}$ Division of Cardiovascular Diseases, Department of Internal Medicine, Mayo Clinic, Rochester, MN, USA and ${ }^{9}$ Division of Endocrinology, Diabetes, Metabolism, and Nutrition, Department of Internal Medicine, Mayo Clinic Rochester, MN, USA

Received: 26 March 2010 Accepted: 17 May 2010

Published: 17 May 2010

\section{References}

1. Nawar EW, Niska RW, Xu J: National Hospital Ambulatory Medical Care Survey: 2005 emergency department summary. Adv Data 2007:1-32.

2. Pope JH, Aufderheide TP, Ruthazer R, Woolard RH, Feldman JA, Beshansky $J R$, Griffith JL, Selker HP: Missed diagnoses of acute cardiac ischemia in the emergency department. New England Journal of Medicine 2000, 342:1163-1170.

3. Kachalia A, Gandhi TK, Puopolo AL, Yoon C, Thomas EJ, Griffey R, Brennan TA, Studdert DM: Missed and delayed diagnoses in the emergency department: a study of closed malpractice claims from 4 liability insurers. Ann Emerg Med 2007, 49:196-205.

4. Pines JM, Szyld D: Risk tolerance for the exclusion of potentially lifethreatening diseases in the ED. Am J Emerg Med 2007, 25:540-544.

5. Fazel R, Krumholz HM, Wang Y, Ross JS, Chen J, Ting HH, Shah ND, Nasir K, Einstein AJ, Nallamothu BK: Exposure to low-dose ionizing radiation from medical imaging procedures. N Engl J Med 2009, 361:849-857.

6. France J, Keen C, Bowyer S: Communicating risk to emergency department patients with chest pain. Emerg Med J 2008, 25:276-278.

7. Jaffe AS, Babuin L, Apple FS: Biomarkers in acute cardiac disease: the present and the future. J Am Coll Cardiol 2006, 48:1-11.

8. Hollander JE, Blomkalns AL, Brogan GX, Diercks DB, Field JM, Garvey JL, Gibler WB, Henry TD, Hoekstra JW, Holroyd BR, Hong Y, Kirk JD, O'Neil BJ, Jackson RE, Aufderheide T, Blomkalns AL, Brogan GX, Christenson J, Collins S, Diercks DB, Fesmire FM, Garvey JL, Green GB, Lindsell CJ, Peacock
WF, Pollack CV, Zalenski R, Multidisciplinary Standardized Reporting Criteria Task Force; Standardized Reporting Criteria Working Group of Emergency Medicine Cardiac Research and Education GroupInternational: Standardized reporting guidelines for studies evaluating risk stratification of emergency department patients with potential acute coronary syndromes. Ann Emerg Med 2004, 44:589-598.

9. Agarwal DA, Subhash CDMN, Church C, Kroning J, Wiebush L, Hess EP: Validation of a Web-based, Real-time Electronic Notification System to Identify Potentially Eligible Subjects for Emergency Department Research Studies. Society for Academic Emergency Medicine Annual Meeting, Pheonix, Arizona, (Abstract) 2010.

10. Mann DM, Ponieman D, Montori VM, Arciniega J, McGinn T: The Statin Choice decision aid in primary care: A randomized trial. Patient Educ Couns 2009.

11. Pencille LJ, Campbell ME, Van Houten HK, Shah ND, Mullan RJ, Swiglo BA, Breslin M, Kesman RL, Tulledge-Scheitel SM, Jaeger TM, Johnson RE, Bartel GA, Wermers RA, Melton LJ, Montori VM: Protocol for the Osteoporosis Choice trial. A pilot randomized trial of a decision aid in primary care practice. Trials 2009, 10:113

12. Montori VM, Breslin M, Maleska M, Weymiller AJ: Creating a conversation: insights from the development of a decision aid. PLoS Med 2007, 4:e233.

13. Breslin M, Mullan RJ, Montori VM: The design of a decision aid about diabetes medications for use during the consultation with patients with type 2 diabetes. Patient Educ Couns 2008, 73:465-472.

14. Hess EP, Wells GA, Jaffe A, Stiell IG: A study to derive a clinical decision rule for triage of emergency department patients with chest pain: design and methodology. BMCEmerg Med 2008, 8:3

15. Lyon R, Morris AC, Caesar D, Gray S, Gray A: Chest pain presenting to the Emergency Department-to stratify risk with GRACE or TIMI? Resuscitation 2007, 74:90-93.

16. Hess EP, Agarwal DA, Chandra S, Murad MH, Erwin PJ, Hollander JE, Montori VM, Stiell IG: Accuracy of the TIMI risk score in emergency department patients with chest pain and possible acute coronary syndrome. Canadian Medical Association Journal 2010 in press.

17. Kline JA, Zeitouni RA, Hernandez-Nino J, Jones AE: Randomized Trial of Computerized Quantitative Pretest Probability in Low-Risk Chest Pain Patients: Effect on Safety and Resource Use. Ann Emerg Med 2009.

18. Kline JA, Johnson CL, Pollack CV Jr, Diercks DB, Hollander JE, Newgard CD, Garvey JL: Pretest probability assessment derived from attribute matching. BMC Med Inform Decis Mak 2005, 5:26.

19. Mitchell AM, Garvey JL, Chandra A, Diercks D, Pollack CV, Kline JA: Prospective multicenter study of quantitative pretest probability assessment to exclude acute coronary syndrome for patients evaluated in emergency department chest pain units. Ann Emerg Med 2006, 47:447.

20. Pocock SJ: Allocation of patients to treatment in clinical trials. Biometrics 1979, 35:183-197.

21. Fergusson D, Aaron SD, Guyatt G, Hebert P: Post-randomisation exclusions: the intention to treat principle and excluding patients from analysis. BMJ 2002, 325:652-654.

22. Nannenga MR, Montori VM, Weymiller AJ, Smith SA, Christianson TJ, Bryant SC, Gafni A, Charles C, Mullan RJ, Jones LA, Bolona ER, Guyatt GH: A treatment decision aid may increase patient trust in the diabetes specialist. The Statin Choice randomized trial. Health Expect 2009, 12:38-44.

23. Jones LA, Weymiller AJ, Shah N, Bryant SC, Christianson TJ, Guyatt GH, Gafni A, Smith SA, Montori VM: Should clinicians deliver decision aids? Further exploration of the statin choice randomized trial results. Med Decis Making 2009, 29:468-474.

24. Legare F, O'Connor AM, Graham ID, Wells GA, Tremblay S: Impact of the Ottawa Decision Support Framework on the agreement and the difference between patients' and physicians' decisional conflict. Med Decis Making 2006, 26:373-390.

25. Ryan RM, Deci EL: Self-determination theory and the facilitation of intrinsic motivation, social development, and well-being. Am Psychol 2000, 55:68-78.

26. Thygesen K, Alpert JS, White HD: Joint ESC/ACCF/AHA/WHF Task Force for the Redefinition of Myocardial Infarction. Universal definition of myocardial infarction. Circulation 2007, 116:1-20.

27. Melton LJ: History of the Rochester Epidemiology Project. Mayo Clin Proc 1996, 71:266-274. 
28. Social Security Administration, Office of Policy. Announcement of Service to Epidemiological Researchers to Provide Vital Status Data on Subjects of Health Research. Federal Register 1998, 63:44942-44944.

29. O'Connor AM, Bennett C, Stacey D, Barry MJ, Col NF, Eden KB, Entwistle V, Fiset V, Holmes-Rovner M, Khangura S, Llewellyn-Thomas H, Rovner DR: Do patient decision aids meet effectiveness criteria of the international patient decision aid standards collaboration? A systematic review and meta-analysis. Med Decis Making 2007, 27:554-574.

30. Weymiller AJ, Montori VM, Jones LA, Gafni A, Guyatt GH, Bryant SC, Christianson TJ, Mullan RJ, Smith SA: Helping patients with type 2 diabetes mellitus make treatment decisions: statin choice randomized trial. Arch Intern Med 2007, 167:1076-1082.

31. Thom DH, Ribisl KM, Stewart AL, Luke DA: Further validation and reliability testing of the Trust in Physician Scale. The Stanford Trust Study Physicians. Med Care 1999, 37:510-517.

32. O'Connor AM: Validation of a decisional conflict scale. Med Decis Making 1995, 15:25-30.

33. Elwyn G, Hutchings H, Edwards A, Rapport F, Wensing M, Cheung WY, Grol R: The OPTION scale: measuring the extent that clinicians involve patients in decision-making tasks. Health Expect 2005, 8:34-42.

34. Mullan RJ, Montori VM, Shah ND, Christianson TJ, Bryant SC, Guyatt GH, Perestelo-Perez LI, Stroebel RJ, Yawn BP, Yapuncich V, Breslin MA, Pencille L, Smith SA: The diabetes mellitus medication choice decision aid: a randomized trial. Arch Intern Med 2009, 169:1560-1568.

35. Harris PA, Taylor R, Thielke R, Payne J, Gonzalez N, Conde JG: Research electronic data capture (REDCap)--a metadata-driven methodology and workflow process for providing translational research informatics support. J Biomed Inform 2009, 42:377-381.

doi: 10.1186/1745-6215-11-57

Cite this article as: Pierce et al., The Chest Pain Choice trial: a pilot randomized trial of a decision aid for patients with chest pain in the emergency department Trials 2010, 11:57

Submit your next manuscript to BioMed Central and take full advantage of:

- Convenient online submission

- Thorough peer review

- No space constraints or color figure charges

- Immediate publication on acceptance

- Inclusion in PubMed, CAS, Scopus and Google Scholar

- Research which is freely available for redistribution

Submit your manuscript at www.biomedcentral.com/submit
C) Biomed Central 